\title{
Los consumidores y la cocreación de valor: el caso de una comunidad de marca virtual ${ }^{*}$
}

\author{
Consumers and Value Co-Creation: The Case of an Online Brand Community
}

\author{
María Cristina Otero-Gómez \\ Magíster en Mercadeo, Universidad de los Llanos, \\ Villavicencio - Colombia, motero@unillanos.edu.co \\ Wilson Giraldo-Pérez \\ Magíster en Mercadeo, Universidad de los Llanos, \\ Villavicencio - Colombia,wgiraldo@unillanos.edu.co
}

\begin{abstract}
Cómo citar / How to cite
Otero-Gómez, M. C., Giraldo-Pérez, W. (2021). Los consumidores y la cocreación de valor: el caso de una comunidad de marca virtual. Revista CEA, v. 7, n. 15, e1847. https://doi.org/10.22430/24223182.1847
\end{abstract}

Recibido: 18 de febrero de 2021

Aceptado: 15 de junio de 2021

\begin{abstract}
Resumen
Este artículo tiene como objetivo analizar la cocreación de valor a partir de los comentarios online generados por el consumidor y su influencia en las intenciones de recompra de una marca. Se trata de un estudio de caso aplicado a una comunidad de marca de vehículos en Colombia ubicada en la red social Facebook. El estudio se fundamenta en la creación y circulación de comentarios positivos por parte del consumidor en relación con su marca, abordando principalmente los conceptos defensa, evangelismo y promoción. Para la realización de este estudio se utilizó un enfoque cuantitativo en el que se procesaron los datos procedentes de 140 encuestas virtuales, sirviéndose del software SmartPLS 3.0 para realizar el análisis factorial y así determinar la validez y confiablidad del instrumento. Posteriormente se usó el modelo de ecuaciones estructurales para corroborar las hipótesis. Los datos revelan que las variables que presentan influencia en las intenciones de recompra son, en su orden: promoción de marca y evangelismo de marca. Por el contrario, la defensa de la marca carece de influencia en el comportamiento posconsumo. Esto sugiere que los consumidores, como miembros de una comunidad, son más proclives a realizar acciones de evangelización y promoción que favorecen la marca, como, por ejemplo: brindar información a otros clientes sobre los aspectos positivos de esta, recomendarla a nuevos consumidores, promover la generación de opiniones positivas tanto online como offline y otros comportamientos que evidencien un elevado
\end{abstract}

\footnotetext{
* Este artículo se deriva del proyecto titulado «Antecedentes y consecuencias del amor a la marca. Análisis en los entornos offline y online» y ha sido avalado por la Universidad de los Llanos y financiado con recursos propios.
} 
nivel de apoyo. De este modo, la promoción requiere de un amplio conocimiento y experiencia con la marca, lo que conlleva a repetir su compra y a adoptar de forma temprana los nuevos lanzamientos para la diversificación de la marca.

Palabras clave: defensa de marca, evangelismo de marca, promoción de marca, intención de recompra, comunidad de marca.

Clasificación JEL: M30, M31, D83.

\title{
Highlights
}

- Los comentarios positivos del consumidor influyen en la intención de recompra.

- El evangelismo y la promoción de marca son antecedentes de la intención de recompra.

- La cocreación de valor por parte del consumidor, impacta su intención de recompra.

\begin{abstract}
This article analyzes value co-creation in online comments posted by consumers and their influence on brand repurchase intention. This applied case study examined a Colombian vehicle brand community on Facebook and investigated the generation and dissemination of positive comments by consumers regarding the brand, especially focusing on the concepts of defense, evangelism, and promotion. This study adopted a quantitative approach, in which the data derived from 140 virtual interviews was processed using SmartPLS software (version 3.0) to conduct a factor analysis and thus determine the validity and reliability of the instrument. Afterward, structural equation modeling was used to test the hypotheses. The data revealed that the variables brand promotion and brand evangelism, in that order, influence repurchase intention. By contrast, brand defense has no influence on post-consumption behavior. This suggests that consumers who are part of a community are more likely to perform evangelization and promotion actions that favor the brand, e.g., sharing information about positive aspects of the brand with other customers, recommending it to new consumers, promoting the generation of positive opinions online and offline, and adopting other behaviors that show a high level of support. Therefore, consumers need to have a vast knowledge of and experience with a brand in order to promote it, which leads to repeat purchases and an early adoption of new releases, which favors brand diversification.
\end{abstract}

Keywords: brand defense, brand evangelism, brand promotion, repurchase intention, brand community.

JEL classification: M30, M31, D83.

\section{Highlights}

- Positive consumer comments influence repurchase intention.

- Brand evangelism and promotion are antecedents of repurchase intention.

- Value co-creation by consumers influences their repurchase intention. 


\section{INTRODUCCIÓN}

En la actualidad, las comunidades de marca virtuales se han convertido en un recurso fundamental para acercar a las empresas con los consumidores (Chen et al., 2021). De este modo, las empresas pueden recibir comentarios oportunos de los clientes sobre los bienes y servicios que tienen disponibles en el mercado, así como acoger sus consejos y aportes tendientes al diseño de nuevas creaciones (Zhao et al., 2019). De forma complementaria, estas comunidades también afianzan los vínculos entre sus integrantes, dado que son un espacio diseñado pare realizar múltiples acciones, entre ellas, hacer que sus miembros se conviertan en proveedores y/o beneficiarios de la información relacionada con su marca focal (Skålén et al., 2015). En este sentido, la información que divulgan las comunidades de marca puede ejercer una influencia importante en los consumidores durante los procesos de compra o recompra de productos y marcas.

A partir del intercambio de información, los miembros de una comunidad virtual pueden asumir diferentes roles como cocreadores de valor (Liu et al., 2020). Por ejemplo, cuando la relación es muy fuerte hace que los consumidores generen contenido online con una alta orientación hacia la defensa de la marca, al tiempo que la recomiendan a clientes potenciales. En otros casos, mediante la emisión de comentarios positivos sobre su marca focal, actúan como evangelistas y promotores que persuaden a nuevos seguidores para que utilicen la marca de interés. De acuerdo con Cossío-Silva et al. (2016), la cocreación de valor tiene la capacidad de impulsar la lealtad de los consumidores, principalmente cuando estos comparten información de la marca con otros, cuando se asesoran mutuamente sobre el uso de esta o cuando brindan retroalimentación para mejorarla. Por tanto, los consumidores que están altamente relacionados o involucrados con su marca demuestran un alto interés de repetir la compra (Hollebeek, 2013).

El intercambio de información se dinamiza gracias al avance de los medios sociales, como es el caso de Facebook, Instagram, YouTube, Twitter, WeChat y TikTok, entre otros, por lo que esta temática ha despertado el interés de los académicos en el sentido de investigar el proceso que impulsa la cocreación de valor a través de las interacciones entre el consumidor y la marca (Cheung et al., 2021). Para efectos de este manuscrito, se ha considerado una comunidad de marca de vehículos en Colombia alojada en el portal de Facebook, debido a que la información que están dispuestos a compartir es para socios y no socios del club. El propósito fundamental de la investigación es indagar cuál de los tres conceptos de cocreación de valor a partir de comentarios, es decir, la defensa de la marca (DM), el evangelismo de marca (EM) o la promoción de la marca (PM), tiene mayor influencia en la intención de recompra (IR) por parte del consumidor. En tal sentido, la pregunta que guía esta investigación es ¿qué elementos de la cocreación de valor relacionados con la generación de comentarios positivos influyen en la intención de recompra de los miembros de una comunidad de marca?

En general, este estudio contribuye a la literatura de marketing al ofrecer un marco integral que demuestra cómo la información emitida y divulgada por los consumidores en el entorno online influye en la cocreación de valor y en el comportamiento posconsumo, concretamente en IR. La estructura de este manuscrito presenta, en la parte inicial, el fundamento teórico y el modelo conceptual, así como las hipótesis que soportan la investigación. Seguidamente se describe la metodología utilizada y luego se exponen los principales resultados. Por último, se discuten las implicaciones para la teoría y la práctica, y en el cierre se detallan las principales conclusiones. 


\section{MARCO TEÓRICO}

Los medios sociales son aplicaciones, plataformas, herramientas web o sistemas tecnológicos en línea que facilitan la colaboración y el intercambio de contenido entre los miembros de la comunidad (Kaplan y Haenlein, 2010; Rubio et al., 2019). Desde el punto de vista de Muniz y O'guinn (2001), estas comunidades se basan en un conjunto estructurado de relaciones sociales entre admiradores de una marca. En tal sentido, existen algunos factores estructurales que posibilitan la creación y el éxito de la comunidad, como son: la cercanía entre los miembros, la frecuencia en la conectividad, la fluidez en la comunicación y el grado de similitud entre los miembros (Lee et al., 2011).

Sumado a esto, el valor de la comunidad de marca virtual se materializa cuando existe la capacidad de involucrar a los consumidores y en mantener las relaciones con estos (Zhao et al., 2019). Aunque no todos los miembros tienen la misma disposición de participar de manera decidida en las actividades de la comunidad (Royo-Vela y Casamassima, 2011), es evidente que interactuar con otros y adquirir información son las dos principales razones por las que los consumidores deciden participar en estos grupos (Brodie et al., 2013).

Las razones anteriores dejan en evidencia que la interacción social es un pilar de la comunidad de marca (Mingione et al., 2020); como reflejo de esta interacción, los investigadores han propuesto un nuevo paradigma de marketing: la cocreación de valor (Prahalad y Ramaswamy, 2004). En el contexto de las comunidades de marca, el valor se manifiesta a través de la promulgación colectiva de prácticas que favorecen la marca, en lugar de limitarse a las prácticas tradicionales de marketing que involucran exclusivamente la díada empresa-consumidor (Schau et al., 2009). Bajo esta premisa, los comportamientos de cocreación se refieren a interacciones en las que participan el consumidor con su marca, aunque también se manifiesta cuando surgen acciones recíprocas entre la marca y otros consumidores, amigos, familiares y otras redes (France et al., 2015).

La cocreación de valor para la marca es un constructo que demanda una serie de comportamientos activos por parte del consumidor (France et al., 2020; Gómez-Bayona et al., 2020), bien sea de manera independiente o como miembro de una comunidad. Los académicos del marketing destacan dos tipos de comportamientos: en primer lugar, están los comportamientos de participación, que se refieren a la búsqueda e intercambio de información; en segundo lugar, se ubican los comportamientos de ciudadanía, en el que sobresale la ayuda a otros consumidores, y la defensa, tanto de la comunidad, como de la marca (Rubio et al., 2020; Yi y Gong, 2013). Adicionalmente, la literatura también refiere que existen otras formas de participación de los consumidores en los procesos de cocreación, como el evangelismo de la marca (Schau et al., 2009; Banda et al., 2020) y la promoción (Jillapalli y Wilcox, 2010).

Para los fines de este manuscrito, DM se define como una manifestación de apoyo a la marca por parte de los consumidores, quienes se encargan de salvaguardar la reputación de la marca y de proteger los intereses de esta (Wilk et al., 2020). Se trata de una forma de participación altamente valorada por las empresas, puesto que los consumidores «se convierten en grandes aliados dispuestos a defender su marca ante el surgimiento de críticas o cuando surgen pronunciamientos negativos de los detractores» (Otero-Gómez y Giraldo-Pérez, 2020, p. 231).

De otro lado, EM va más allá de la defensa, puesto que no solamente busca mantener la integridad de la marca, sino que trata de reclutar a otros consumidores para que se conviertan en seguidores, 
compradores o usuarios de la marca preferida por el consumidor inicial. Matzler et al. (2007) la definen como el acto de "difundir opiniones positivas y tratar fervientemente de convencer o persuadir a otros para que se comprometan con la misma marca» (p. 27).

En cuanto a PM, esta se define como el grado en el que los consumidores están dispuestos a dedicar tiempo y esfuerzo para recomendar y apoyar activamente su marca en relación con la competencia (Jillapalli y Wilcox, 2010). Esto sugiere que, cuando los consumidores desarrollan fuertes vínculos con una marca, son más propensos a difundir comentarios positivos sobre ella (Otero-Gómez y GiraldoPérez, 2019).

Estos conceptos son significativos a la hora de desarrollar estrategias de comunicación tendientes a aumentar el valor, tanto para la marca, como para los consumidores, debido a la capacidad que tienen para influir en las percepciones de otros individuos en relación con la marca, los bienes, los servicios y la organización en general (Singh y Trinchetta, 2020). La literatura sobre marketing demuestra que tanto las características de la información generada a través del boca a boca electrónico como el comportamiento del consumidor hacia esta información, tienen un impacto positivo en el interés de adquirir bienes y servicios (Nuriman Izudin et al., 2020).

Sumado a lo anterior, entre las variables de resultado que se generan a partir de la cocreación de valor, la literatura identifica a IR como un concepto que cada vez cobra mayor importancia, tanto en el entorno empresarial, como en el académico. De este modo, IR se refiere al compromiso de repetir las compras de un proveedor de servicios en particular (Su et al., 2016). También refleja el deseo de actualizar, cambiar o comprar nuevos productos de la misma marca (Herjanto y Amin, 2020). En la actualidad se ha demostrado la existencia de respuestas favorables hacia la marca y su éxito en el mercado, tras confirmar la relación entre la cocreación de valor desde la perspectiva del consumidor y la intención de recompra (Hoyer et al., 2010; Alarcón López et al., 2017). Basados en esta fundamentación teórica se desprende el modelo de investigación aquí presentado (ver Figura 1).

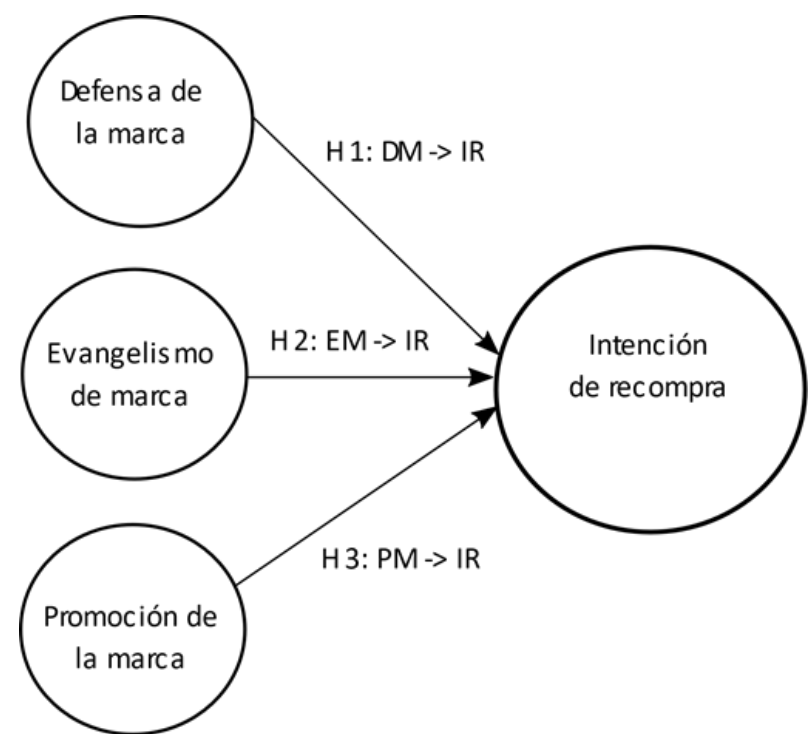

Figura 1. Modelo de investigación

Figure 1. Research model

Fuente: elaboración propia. 
A partir de lo anterior, este manuscrito plantea las siguientes hipótesis:

H1. La defensa de la marca influye positivamente en la intención de recompra.

H2. El evangelismo de marca influye positivamente en la intención de recompra.

H3. La promoción de la marca influye positivamente en la intención de recompra.

\section{METODOLOGÍA}

A continuación, se detallan los procedimientos y las técnicas utilizadas durante la recolección de datos junto con el procesamiento del análisis factorial exploratorio de las ecuaciones estructurales.

\section{Contexto de estudio}

La investigación se enfocó en analizar una comunidad de marca de vehículos en Colombia. Antes de iniciar el trabajo de campo se hizo un análisis de contenido de los comentarios que los miembros de la comunidad realizan a través de Facebook, con el fin de establecer la viabilidad del estudio. La razón por la que se seleccionó tanto a la marca como a la comunidad es porque sus miembros expresaron una gran disposición para responder la encuesta, dado que para ellos es una oportunidad de demostrar su orgullo como usuarios de la marca, aspecto que está ampliamente respaldado por la teoría asociada al reconocimiento social.

\section{Muestra}

Para el desarrollo de esta investigación cuantitativa se consideró como nicho objetivo a los propietarios de vehículos con edades entre los 18 y 64 años. Se optó por la implementación de un muestreo por conveniencia. Se encuestaron, de manera virtual, a 140 individuos que fueron conectados a través del método de muestreo de bola de nieve. El perfil sociodemográfico indica que el 91\% pertenece al segmento masculino, y el 9\% al femenino. La media de la edad es de 43.4 años, y su distribución por edades es la siguiente: el 12\% concentra a las personas entre 18 y 30 años; el $20 \%$ abarca a los usuarios entre 30 y 40 años; el $42 \%$ lo conforman individuos entre 40 y 50 años; y, el $26 \%$ corresponde a mayores de 50 años. Estos datos reflejan que aproximadamente el $70 \%$ de la muestra es mayor de 40 años. En una etapa previa a la recolección de la información se explicó a los participantes que se trata de una encuesta completamente anónima, por lo que no se recoge ninguna información que pueda identificarlos individualmente. En todos los casos se atendieron los protocolos establecidos por el Comité de Bioética de la Universidad de los Llanos, institución que avala esta investigación.

\section{Diseño de la investigación}

Para el análisis de las valoraciones de los miembros de la comunidad se diseñó un cuestionario estructurado con el fin de reunir la información necesaria para alcanzar los objetivos del estudio. Los ítems utilizados han sido testeados en investigaciones previas, por lo que en este estudio se tradujeron y adaptaron las medidas con sus respectivos autores, así: DM (Dalman et al. 2019); EM (Matzler et al., 2007), PM (Kim et al., 2002; Yi y Gong, 2013; Shimul et al., 2019) y en la variable de resultado IR (Chiu et al., 2009; Chai et al., 2015). En el Anexo 1 se presenta el instrumento utilizado 
para la recolección de los datos. En todos los ítems los participantes informaron su nivel de identificación para cada declaración en una escala Likert de 7 puntos ( 1 = Totalmente en desacuerdo y 7 = Totalmente de acuerdo). Para el procesamiento y análisis de los datos se utilizó el software SmartPLS 3.0.

\section{Análisis factorial}

Para determinar la validez convergente del instrumento definitivo utilizado en la recolección de la información, se analizó la carga que cada ítem refleja del constructo (ver Tabla 1). Los resultados indican que los valores correspondientes a cada ítem presentan cargas que varían desde 0.717 hasta 0.950 . Asimismo, la fiabilidad del ítem varía desde 0.515 hasta 0.903 ; y la varianza media extraída se encuentra entre 0.711 y 0.890 . Los resultados de las pruebas de validez discriminante son presentados en las tablas subsiguientes, así se presentan por separado el criterio de cargas cruzadas (ver tabla 2) y en conjunto, las pruebas Fornell-Larcker y ratio Heterotreid/Monotreid (ver tabla 3).

Tabla 1. Cargas y varianza media extraída para la validación convergente

Table 1. Loadings and average variance extracted for convergent validity

\begin{tabular}{|c|c|c|c|c|c|c|}
\hline ĺtom & Carga & Estadístico & $P$ value & Fiabilidad & Varianza media & Cumple \\
\hline Item & $>0.7$ & $\mathrm{t}$ & $<0.05$ & ítem >0.5 & extraída > 0.5 & criterios \\
\hline Ítem 1DM & 0.939 & 45.545 & 0.000 & 0.882 & \multirow{3}{*}{0.890} & Sí \\
\hline Ítem 2DM & 0.950 & 72.844 & 0.000 & 0.903 & & Sí \\
\hline Ítem 3DM & 0.941 & 47.992 & 0.000 & 0.885 & & Sí \\
\hline Ítem 1EM & 0.809 & 20.947 & 0.000 & 0.654 & \multirow{5}{*}{0.711} & Sí \\
\hline Ítem 2EM & 0.924 & 59.505 & 0.000 & 0.854 & & Sí \\
\hline Ítem 3EM & 0.889 & 28.256 & 0.000 & 0.790 & & Sí \\
\hline Ítem 4EM & 0.862 & 20.852 & 0.000 & 0.743 & & Sí \\
\hline Ítem 5EM & 0.717 & 10.420 & 0.000 & 0,515 & & Sí \\
\hline Ítem 1PM & 0.922 & 41.836 & 0.000 & 0.850 & \multirow{5}{*}{0.859} & Sí \\
\hline Ítem 2PM & 0.934 & 40.813 & 0.000 & 0.873 & & Sí \\
\hline Ítem 3PM & 0.918 & 33.372 & 0.000 & 0.842 & & Sí \\
\hline Ítem 4PM & 0.940 & 55.791 & 0.000 & 0.884 & & Sí \\
\hline Ítem 5PM & 0.919 & 38.159 & 0.000 & 0.845 & & Sí \\
\hline Ítem 1IR & 0.920 & 48.947 & 0.000 & 0.846 & \multirow{4}{*}{0.763} & Sí \\
\hline Ítem 2IR & 0.923 & 37.541 & 0.000 & 0.853 & & Sí \\
\hline Ítem 3IR & 0.745 & 11.684 & 0.000 & 0.555 & & Sí \\
\hline Ítem 4IR & 0.895 & 20.342 & 0.000 & 0.800 & & Sí \\
\hline
\end{tabular}


Tabla 2. Cargas cruzadas para la validación discriminante

Table 2. Cross loadings for discriminant validity

\begin{tabular}{cccccc}
\hline Ítem & $\begin{array}{c}\text { Defensa de la } \\
\text { marca }\end{array}$ & $\begin{array}{c}\text { Evangelismo } \\
\text { de marca }\end{array}$ & $\begin{array}{c}\text { Promoción } \\
\text { de la marca }\end{array}$ & $\begin{array}{c}\text { Intención de } \\
\text { recompra }\end{array}$ & $\begin{array}{c}\text { Cumple } \\
\text { criterios }\end{array}$ \\
\hline Ítem 1DM & 0.939 & 0.742 & 0.805 & 0.498 & Sí \\
\hline Ítem 2DM & 0.950 & 0.734 & 0.803 & 0.583 & Sí \\
\hline Ítem 3DM & 0.941 & 0.667 & 0.799 & 0.543 & Sí \\
\hline Ítem 1EM & 0.525 & 0.809 & 0.471 & 0.405 & Sí \\
\hline Ítem 2EM & 0.746 & 0.924 & 0.686 & 0.542 & Sí \\
\hline Ítem 3EM & 0.726 & 0.889 & 0.705 & 0.493 & Sí \\
\hline Ítem 4EM & 0.644 & 0.862 & 0.644 & 0.515 & Sí \\
\hline Ítem 5EM & 0.506 & 0.717 & 0.463 & 0.332 & Sí \\
\hline Ítem 1PM & 0.791 & 0.694 & 0.922 & 0.573 & Sí \\
\hline Ítem 2PM & 0.812 & 0.700 & 0.934 & 0.536 & Sí \\
\hline Ítem 3PM & 0.768 & 0.702 & 0.918 & 0.576 & Sí \\
\hline Ítem 4PM & 0.804 & 0.616 & 0.940 & 0.645 & Sí \\
\hline Ítem 5PM & 0.762 & 0.613 & 0.919 & 0.565 & Sí \\
\hline Ítem 1IR & 0.566 & 0.550 & 0.627 & 0.920 & Sí \\
\hline Ítem 2IR & 0.552 & 0.555 & 0.609 & 0.923 & Sí \\
\hline Ítem 3IR & 0.427 & 0.348 & 0.416 & 0.745 & Sí \\
\hline Ítem 4IR & 0.447 & 0.440 & 0.504 & 0.895 & Sí \\
\hline & & Fuente: elaboración propia. & &
\end{tabular}

Tabla 3. Prueba Fornell-Larcker y ratio Heterotreid/Monotreid para la validación discriminante Table 3. Fornell-Larcker test and heterotrait-monotrait ratio for discriminant validity

\begin{tabular}{cccccc}
\hline & $\begin{array}{c}\text { Defensa de } \\
\text { la marca }\end{array}$ & $\begin{array}{c}\text { Evangelismo } \\
\text { de marca }\end{array}$ & $\begin{array}{c}\text { Promoción de } \\
\text { la marca }\end{array}$ & $\begin{array}{c}\text { Intención de } \\
\text { recompra }\end{array}$ & $\begin{array}{c}\text { Cumple } \\
\text { criterios }\end{array}$ \\
\hline Defensa de la marca & $(0.943)$ & 0.815 & 0.896 & 0.620 & Sí \\
\hline Evangelismo de marca & 0.757 & $(0.843)$ & 0.762 & 0.595 & Sí \\
\hline Promoción de la marca & 0.850 & 0.716 & $(0.927)$ & 0.664 & Sí \\
\hline Intención de recompra & 0.576 & 0.552 & 0.627 & $(0.873)$ & Sí \\
\hline
\end{tabular}

Fuente: elaboración propia.

* En la diagonal, dentro del paréntesis, la raíz cuadrada de la varianza media extraída de las dimensiones; debajo de la diagonal, los resultados de las correlaciones con otras dimensiones en el modelo; y sobre la diagonal, los coeficientes del ratio Heterotrait Monotrait.

Finalmente, la consistencia interna se analizó a partir del índice de fiabilidad compuesta y el alfa de Cronbach, cuyos valores deben superar el umbral de 0.7 (ver Tabla 4). 
Tabla 4. Índice de fiabilidad compuesta y alfa de Cronbach para la consistencia interna Table 4. Composite reliability index and Cronbach's alpha for internal consistency

\begin{tabular}{cccc}
\hline & $\begin{array}{c}\text { Índice de Fiabilidad } \\
\text { Compuesta } \\
>0.7\end{array}$ & $\begin{array}{c}\text { Alfa de } \\
\text { Cronbach } \\
>0.7\end{array}$ & $\begin{array}{c}\text { Cumple } \\
\text { criterios }\end{array}$ \\
\hline Defensa de la marca & 0.960 & 0.938 & Sí \\
\hline Evangelismo de marca & 0.924 & 0.897 & Sí \\
\hline Promoción de la marca & 0.968 & 0.959 & Sí \\
\hline Intención de recompra & 0.928 & 0.896 & Sí \\
\hline \multicolumn{5}{c}{ Fuente: elaboración propia. }
\end{tabular}

Una vez comprobado el cumplimiento satisfactorio de los resultados esperados en el análisis factorial, se garantizó la validez de contenido, por lo que se procedió a realizar el análisis estructural con el fin de comprobar las hipótesis planteadas, cuya significancia se determinó mediante un proceso de remuestreo (bootstrapping) de 5000 submuestras utilizadas para evaluar la precisión de las estimaciones de los mínimos cuadrados parciales.

\section{RESULTADOS}

En este estudio se plantearon tres hipótesis que buscaban explicar la influencia positiva de la cocreación de valor a partir de los comentarios sobre la intención de repetir la compra de productos relacionados con la marca focal (ver Figura 2). En su respectivo orden se analizó la relación existente entre la DM e IR, el EM e IR, y finalmente, PM e IR. En los resultados del análisis estructural se presentan los coeficientes de regresión estandarizados (path) y la significancia de cada hipótesis (ver Tabla 5).

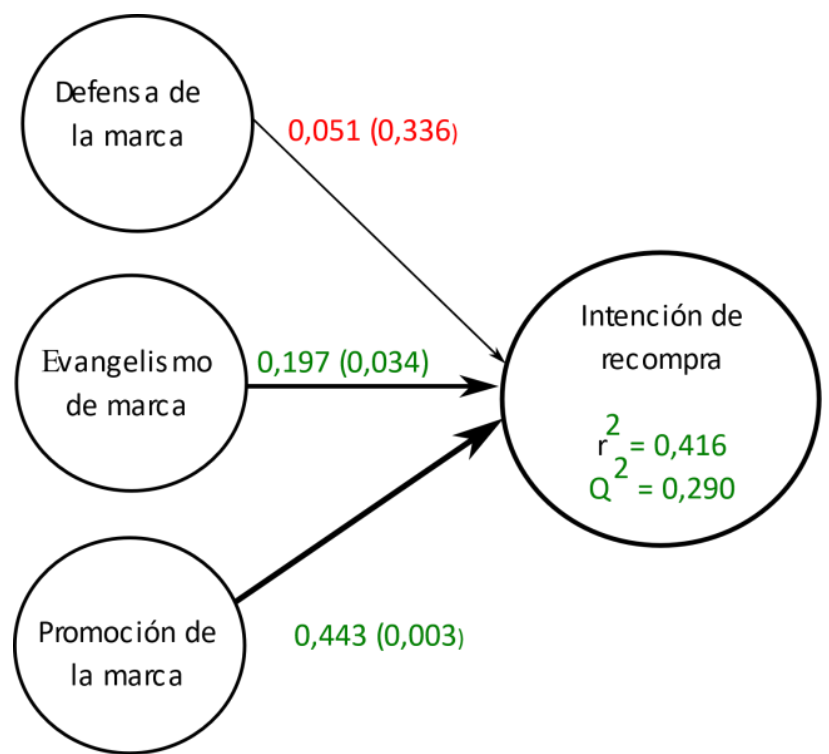

Figura 2. Modelo estructural de salida

Figure 2. Resulting structural model

Fuente: elaboración propia. 
Tabla 5. Validación de hipótesis

Table 5. Hypothesis testing

\begin{tabular}{ccccc}
\hline & Path & Estadísticos t & P Valores & Decisión \\
\hline H1 Defensa de la marca -> Intención de recompra & 0.051 & 0.342 & 0.366 & Rechazada \\
\hline $\begin{array}{c}\text { H2 Evangelismo de marca -> Intención de } \\
\text { recompra }\end{array}$ & 0.197 & 1.822 & 0.034 & Aceptada \\
\hline $\begin{array}{c}\text { H3 Promoción de la marca -> Intención de } \\
\text { recompra }\end{array}$ & 0.443 & 2.706 & 0.003 & Aceptada \\
\hline
\end{tabular}

Fuente: elaboración propia.

Los resultados demuestran que existe una influencia positiva tanto de PM como de EM sobre IR. Por el contrario, no se pudo confirmar la influencia de DM en el comportamiento poscompra estudiado, es decir, sobre IR.

La relevancia predictiva del modelo estructural (ver Tabla 6) se demuestra a partir de los valores de la cantidad de varianza de IR explicada por las variables predictoras $\left(R^{2}\right)$ y mediante el desarrollo del procedimiento blindfolding que establece la relevancia predictiva con validación cruzada del modelo de trayectoria $\left(Q^{2}\right)$.

Tabla 6. Relevancia predictiva del modelo estructural

Table 6. Predictive relevance of the structural model

\begin{tabular}{ccccccc}
\hline & $\mathrm{R}^{2}$ & Estadísticos t & $\mathrm{P}$ Valores & SSO & SSE & $\mathrm{Q}^{2}=(1-(\mathrm{SSE} / \mathrm{SSO}))$ \\
\hline $\begin{array}{c}\text { Intención de } \\
\text { recompra }\end{array}$ & 0.416 & 4.282 & 0.000 & 560.000 & 397.832 & 0.290 \\
\hline
\end{tabular}

Fuente: elaboración propia.

\section{DISCUSIÓN}

Esta investigación tuvo como objetivo analizar la cocreación de valor en una comunidad de marca virtual a partir de los comentarios online generados por el consumidor y su influencia en las intenciones de recompra de una marca. Concretamente, el estudio se enfoca en identificar si DM, EM y PM influyen positivamente en IR desde el punto de vista de los miembros de la comunidad de marca. Tras la revisión de literatura realizada, se pudo establecer que es la primera investigación que integra estos conceptos y los relaciona con las intenciones de comportamientos posconsumo. Los hallazgos contribuyen a la ampliación del debate académico sobre el rol que desempeñan los consumidores como cocreadores de valor y su importancia para las marcas y, por ende, para las empresas. Por tanto, proporciona una respuesta al llamado de los académicos sobre la comprensión de los efectos de los comentarios positivos realizados a través del boca a boca electrónico (OteroGómez y Giraldo-Pérez, 2019), al tiempo que profundiza en las consecuencias de las interacciones entre el consumidor y la marca (Cheung et al., 2021).

Basados en la premisa de que los consumidores protegen los intereses de la marca hasta el punto de llegar a convertirse en sus defensores (Wilk et al., 2020), se propuso que DM, entendida como una de las formas cocreación de valor, tiene la capacidad de impulsar la lealtad de los consumidores (Cossío-Silva et al., 2016). A pesar de que Marticotte et al. (2016) argumentan que los comentarios 
positivos sobre una marca son una respuesta de los usuarios y miembros de la comunidad para proteger su propia autoestima, principalmente cuando su marca es insultada, esta investigación demostró que DM no es suficiente para conducir a IR. Este resultado contradice los hallazgos de Coelho et al., (2019), quienes afirman que cuando los consumidores son leales a una marca, pueden adoptar comportamientos defensivos positivos que los conduce al rechazo de otras marcas, incluso cuando estas poseen características superiores en comparación a las de su marca predilecta.

De un modo opuesto, el análisis empírico revela los resultados positivos entre EM e IR. Estudios anteriores mencionan que las marcas adquieren el estatus de "sagrada» y se evangelizan cuando existe un proceso de cocreación comunitario en el que interactúan el «consumidor-marca-otro consumidor» (Wang et al., 2019). Por tanto, esta investigación valida el marco propuesto y confirman los hallazgos de otros investigadores. Específicamente, apoya el planteamiento de Becerra y Badrinarayanan (2013), quienes argumentan que los evangelistas no solo difunden referencias positivas sobre la marca y convencen a otros sobre una marca focal, sino que demuestran su compromiso realizando compras continuas.

Esta investigación también brinda respaldo al postulado de que PM predice la variable de resultado IR. La información derivada de la comunicación consumidor-consumidor en las comunidades en línea juega un papel importante en las decisiones de compra, de manera que la información persuasiva en este entorno es crucial (Zhu et al., 2016). Por consiguiente, este estudio destaca la relevancia de los promotores que dedican tiempo y esfuerzo para recomendar su marca (Jillapalli y Wilcox, 2010) a otros consumidores, mediante declaraciones en línea que reflejan el conocimiento específico y profundo de esta (Wilk et al., 2020). Las actividades de promoción tienen doble efecto en las intenciones de continuar realizando compras de productos de la marca de interés. Por un lado, PM influye en los usuarios en general porque estos buscan calidad en la información y credibilidad de la fuente, cuando se enfrentan a las decisiones de consumo (Zhu et al., 2016). Por otro, para que un consumidor se convierta en promotor, debe contar con un conocimiento previo sobre la marca (Wilk et al., 2020) y la convicción de que su uso es bueno tanto para ellos como para los demás consumidores; de esta forma se mantiene latente la intención de repetir la compra de los productos actuales, junto al interés de adoptar los nuevos lanzamientos de la diversificación de la marca (Keller, 1993).

Con respecto a las implicaciones teóricas, la presente investigación profundiza en la línea ya establecida que tienen las comunicaciones informales online y offline y su efecto en el comportamiento del consumidor. Aunque previamente se ha analizado de forma genérica el concepto del boca a boca electrónico y sus efectos en la lealtad, este trabajo toma constructos de la literatura actual asociados a los comentarios positivos y los integra a la teoría sobre cocreación de valor, extendiendo sus efectos a los comportamientos de respuesta de los miembros de una comunidad de marca virtual.

Los hallazgos anteriores ilustran el poder que tienen los consumidores en la cocreación de valor, principalmente en la capacidad de influir en comportamientos asociados al deseo de actualizar, cambiar o comprar nuevos productos de la misma marca (Herjanto y Amin, 2020). En este sentido, este artículo ofrece algunas contribuciones para los gerentes comerciales. Primero, se deben fortalecer las relaciones entre las empresas y las comunidades de marca brindando información actualizada sobre las novedades de los productos. Segundo, deben fomentar el diálogo con los 
defensores de la marca para que estos miembros de la comunidad trasciendan su rol y se conviertan en evangelizadores o promotores; así, el comportamiento de ciudadanía contribuye al desarrollo de la marca dinamizando los procesos de recompra. Por último, los responsables del área de marketing pueden realizar concursos, trivias, retos y demás eventos que promuevan la marca, con el fin de estimular el comportamiento de participación de los consumidores.

De forma general, el modelo propuesto en esta investigación, que consta de tres variables cocreadoras de valor, tiene relevancia predictiva y es capaz de explicar, en un nivel moderado, la varianza de la variable conductual de respuesta IR. En lo particular, se demuestra la influencia de respuesta de EM y PM sobre IR, lo que da lugar a la posibilidad de extender este estudio a otras comunidades de marca establecidas para otros tipos de bienes o servicios que permita diferenciar usos por género, o con otros segmentos poblacionales.

\section{CONCLUSIONES}

Los procesos actuales de relacionamiento con los consumidores, y de estos con las marcas, se encuentran inmersos en una avalancha de comentarios que se multiplican rápidamente gracias a las tecnologías de la información y las comunicaciones. Estos comportamientos deben alentar a las empresas a prestar atención a los rumores, las críticas y los ataques de sus detractores, aunque también deben reconocer el esfuerzo que realizan otros consumidores cocreando valor mediante la defensa, la evangelización y la promoción de sus marcas.

Este trabajo evidenció la influencia positiva que tienen dos de las tres formas de comunicación estudiadas sobre la intención de recompra de productos para los miembros de una comunidad de marca en Colombia. Específicamente demostró que la defensa de marca mediante comentarios no es suficiente para que un usuario continúe comprando productos. Por el contrario, realizar comentarios positivos que buscan atraer a otras personas hacia los productos y/o marcas que el consumidor actual usa, apoya y recomienda activamente en los entornos online y offline, alienta las intenciones de permanecer leales a la marca.

\section{CONFLICTOS DE INTERÉS}

Los autores declaran que no presentan conflictos de interés financiero, profesional o personal que pueda influir de forma inapropiada en los resultados obtenidos o las interpretaciones propuestas.

\section{CONTRIBUCIÓN DE AUTORES}

Para el desarrollo de este proyecto todos los autores han realizado una contribución significativa especificada a continuación:

María Cristina Otero-Gómez: revisión de la literatura científica, traducción y ajuste de los ítems existentes en la literatura científica, redacción del manuscrito y coordinación del trabajo de campo.

Wilson Giraldo-Pérez: responsable de los aspectos metodológicos, traducción y ajuste de los ítems existentes en la literatura científica, además de procesar y analizar los datos de la investigación. 


\section{REFERENCIAS}

Alarcón López, R., Ruiz de Maya, S., López López. I. (2017). Sharing co-creation experiences contributes to consumer satisfaction. Online Information Review, v. 41, n. 7, 969-984. https://doi.org/10.1108/OIR-09-2016-0267

Banda, A., Pritchard, M., Nhedzi, A. (2020). Brand innovation and value-creating practices of the city of Johannesburg's Twitter community. Communicatio, v. 46, n. 1, 87-112. https://doi.org/10.1080/02500167.2019.1700295

Becerra, E. P., Badrinarayanan, V. (2013). The influence of brand trust and brand identification on brand evangelism. Journal of Product \& Brand Management, v. 22, n. 5/6, 371-383. https://doi.org/10.1108/JPBM-09-2013-0394

Brodie, R. J., Ilic, A., Juric, B., Hollebeek, L. (2013). Consumer engagement in a virtual brand community: an exploratory analysis. Journal of Business Research, v. 66, n. 1, 105-114. https://doi.org/10.1016/i.jbusres.2011.07.029

Chai, J. C. Y., Malhotra, N. K., Alpert, F. (2015). A two-dimensional model of trust-value-loyalty in service relationships. Journal of Retailing and Consumer Services, v. 26, 23-31. https://doi.org/10.1016/j.jretconser.2015.05.005

Chen, L., Yuan, L., Zhu, Z. (2021). Empirical study of consumer participation motivation in value cocreation within cultural and creative virtual brand communities. Asia Pacific Journal of Marketing and Logistics, v. 33, n. 7, 1630-1647. https://doi.org/10.1108/APJML-05-2020-0363

Cheung, M. L., Pires, G., Rosenberger, P. J., Leung, W., Ting, H. (2021). Investigating the role of social media marketing on value co-creation and engagement: An empirical study in China and Hong Kong. Australasian Marketing Journal (AMJ), v. 29, n. 2. https://doi.org/10.1016/i.ausmj.2020.03.006

Chiu, C., Chang, C., Cheng, H., Fang, Y. (2009). Determinants of customer repurchase intention in online shopping. Online Information Review, v. 33, n. 4, 761-784.

https://doi.org/10.1108/14684520910985710

Coelho, A., Bairrada, C., Peres, F. (2019). Brand communities' relational outcomes, through brand love. Journal of Product \& Brand Management, v. 28, n. 2, 154-165. https://doi.org/10.1108/JPBM-09-2017-1593

Cossío-Silva, F. J., Revilla-Camacho, M. A., Vega-Vázquez, M., Palacios-Florencio, B. (2016). Value co-creation and customer loyalty. Journal of Business Research, v. 69, n. 5, 1621-1625. https://doi.org/10.1016/j.jbusres.2015.10.028 
Dalman, M. D., Buche, M. W., Min, J. (2019). The differential influence of identification on ethical judgment: The role of brand love. Journal of Business Ethics, v. 158, n. 3, 875-891. https://doi.org/10.1007/s10551-017-3774-1

France, C., Grace, D., Lo lacono, J., Carlini, J. (2020). Exploring the interplay between customer perceived brand value and customer brand co-creation behaviour dimensions. Journal of brand Management, v. 27, 466-480. https://doi.org/10.1057/s41262-020-00194-7

France, C., Merrilees, B., Miller, D. (2015). Customer brand co-creation: a conceptual model. Marketing Intelligence \& Planning, v. 33, n. 6, 848-864.

https://doi.org/10.1108/MIP-06-2014-0105

Gómez-Bayona, L., Londoño-Montoya, E., Mora-González, B. (2020). Modelos de capital intelectual a nivel empresarial y su aporte en la creación de valor. Revista CEA, v. 6, n. 11, 165-184. https://doi.org/10.22430/24223182.1434

Herjanto, H., Amin, M. (2020). Repurchase intention: the effect of similarity and client knowledge. International Journal of Bank Marketing, v. 38, n. 6, 1351-1371. https://doi.org/10.1108/IJBM-03-2020-0108

Hollebeek, L. D. (2013). The customer engagement/value interface: An exploratory investigation. Australasian Marketing Journal, v. 21, n. 1, 17-24. https://doi.org/10.1016/j.ausmj.2012.08.006

Hoyer, W. D., Chandy, R., Dorotic, M., Krafft, M., Singh, S. S. (2010). Consumer co-creation in new product development. Journal of Service Research, v. 13, n. 3, 283-296. https://doi.org/10.1177/1094670510375604

Jillapalli, R. K., Wilcox, J. B. (2010). Professor brand advocacy: do brand relationships matter? Journal of Marketing Education, v. 32, n. 3, 328-340. https://doi.org/10.1177\%2F0273475310380880

Kaplan, A. M., Haenlein, M. (2010). Users of the world, unite! The challenges and opportunities of social media. Business Horizons, v. 53, n. 1, 59-68. https://doi.org/10.1016/i.bushor.2009.09.003

Keller, K. L. (1993). Conceptualizing, measuring, and managing customer-based brand equity. Journal of Marketing, v. 57, n. 1, 1-22. https://doi.org/10.2307/1252054

Kim, Ch. K., Han, D., Park, S. (2002). The effect of brand personality and brand identification on brand loyalty: Applying the theory of social identification. Japanese Psychological Research, v. 43, n. 4, 195-206. https://doi.org/10.1111/1468-5884.00177

Lee, H. J., Lee, D., Taylor, C. R., Lee, J. (2011). Do online brand communities help build and maintain relationships with consumers? A network theory approach. Journal of Brand Management, v. 19, 213-227. https://doi.org/10.1057/bm.2011.33 
Liu, S., Xiao, W., Fang, C., Zhang, X., Lin, J. (2020). Social support, belongingness, and value cocreation behaviors in online health communities. Telematics and Informatics, v. 50, 101398. https://doi.org/10.1016/i.tele.2020.101398

Marticotte, F., Arcand, M., Baudry, D. (2016). The impact of brand evangelism on oppositional referrals towards a rival brand. Journal of Product \& Brand Management, v. 25, n. 6, 538549. https://doi.org/10.1108/JPBM-06-2015-0920

Matzler, K., Pichler, E., Hemetsberger, A. (2007). Who is spreading the word? The positive influence of extraversion on consumer passion and brand evangelism. American Marketing Association, v. 18, 25-32. $\underline{\text { URL }}$

Mingione, M., Cristofaro, M., Mondi, D. (2020). 'If I give you my emotion, what do I get?' Conceptualizing and measuring the co-created emotional value of the brand. Journal of Business Research, v. 109, 310-320. https://doi.org/10.1016/j.jbusres.2019.11.071

Muniz, A. M., O'guinn, T. C. (2001). Brand community. Journal of Consumer Research, v. 27, n. 4, 412-432. https://doi.org/10.1086/319618

Nuriman Izudin, A., Ruswanti, E., Unggul Januarko, M. (2020). The Effect of YouTube eWOM on Consumer Buying Interest. Revista CEA, v. 6, n. 12, 167-179. https://doi.org/10.22430/24223182.1618

Otero-Gómez, M. C., Giraldo-Pérez, W. (2019). Antecedentes y consecuencias del valor de marca. Un estudio centrado en los consumidores jóvenes. Suma de Negocios, v. 10, n. 23, 81-88. https://doi.org/10.14349/sumneg/2019.V10.N23.A1

Otero-Gómez, M. C., Giraldo-Pérez, W. (2020). Incidencia de las actitudes cognitivas y afectivas de los consumidores en la defensa de una marca local. Revista de Investigación, Desarrollo e Innovación, v. 10, n. 2, 225-236. https://doi.org/10.19053/20278306.v10.n2.2020.10627

Prahalad, C. K., Ramaswamy, V. (2004). Co-creating unique value with customers. Strategy \& Leadership, v. 32, n. 3, 4-9. https://doi.org/10.1108/10878570410699249

Royo-Vela. M., Casamassima, P. (2011). The influence of belonging to virtual brand communities on consumers' affective commitment, satisfaction and word-of-mouth advertising: the Zara case. Online Information Review, v. 35, n. 4, 517-542. https://doi.org/10.1108/14684521111161918

Rubio, N., Villaseñor, N., Yagüe, M. (2020). Value co-creation in third-party managed virtual communities and brand equity. Frontiers in Psychology, v. 11, 927. https://doi.org/10.3389/fpsyg.2020.00927 
Rubio, N., Villaseñor, N., Yague, M. J. (2019). Does use of different platforms influence the relationship between cocreation value-in-use and participants' cocreation behaviors? An application in third-party managed virtual communities. Complexity, v. 2019. https://doi.org/10.1155/2019/7562903

Schau, H. J., Muñiz, A. M., Arnould, E. J. (2009). How brand community practices create value. Journal of Marketing, v. 73, n. 5, 30-51. https://doi.org/10.1509/jmkg.73.5.30

Shimul, A. S., Phau, I., Lwin, M. (2019). Conceptualising luxury brand attachment: scale development and validation. Journal of Brand Management, v. 26, 675-690. https://doi.org/10.1057/s41262-019-00158-6

Singh, R., Trinchetta, G. G. (2020). Community connections: advocating for libraries through effective brand advocacy. Public Library Quarterly, v. 39, n. 4, 295-309. https://doi.org/10.1080/01616846.2019.1613626

Skålén, P., Pace, S. Cova, B. (2015). Firm-brand community value co-creation as alignment of practices. European Journal of Marketing, v. 49, n. 3/4, 596-620. https://doi.org/10.1108/EJM-08-2013-0409

Su, L., Swanson, S. R., Chinchanachokchai, S., Hsu, M. K., Chen, X. (2016). Reputation and intentions: The role of satisfaction, identification, and commitment. Journal of Business Research, v. 69, n. 9, 3261-3269. https://doi.org/10.1016/j.jbusres.2016.02.023

Wang, C. L., Sarkar, J. G., Sarkar, A. (2019). Hallowed be thy brand: Measuring perceived brand sacredness. European Journal of Marketing, v. 53, n. 4, 733-757. https://doi.org/10.1108/EJM-08-2017-0551

Wilk, V., Soutar, G. N., Harrigan, P. (2020). Online brand advocacy (OBA): the development of a multiple item scale. Journal of Product \& Brand Management, v. 29, n. 4, 415-429. https://doi.org/10.1108/JPBM-10-2018-2090

Yi, Y., Gong, T. (2013). Customer value co-creation behavior: Scale development and validation. Journal of Business Research, v. 66, n. 9, 1279-1284. https://doi.org/10.1016/i.jbusres.2012.02.026

Zhao, Y., Chen, Y. P., Zhou, R., Ci, Y. (2019). Factors influencing customers' willingness to participate in virtual brand community's value co-creation: The moderating effect of customer involvement. Online Information Review, v. 43, n. 3, 440-461. https://doi.org/10.1108/OIR-08-2017-0232

Zhu, D. H., Chang, Y., Luo, J. J. (2016). Understanding the influence of C2C communication on purchase decision in online communities from a perspective of information adoption model. Telematics and Informatics, v. 33, n. 1, 8-16.

https://doi.org/10.1016/i.tele.2015.06.001 
ANEXO 1

\begin{tabular}{|c|c|}
\hline Constructo (Autor) & ítem \\
\hline $\begin{array}{l}\text { Defensa de la marca } \\
\text { (Dalman et al., 2019) }\end{array}$ & $\begin{array}{l}\text { 1. Defendería a la marca XXX en una conversación } \\
\text { 2. Protegería a la marca XXX en una conversación } \\
\text { 3. Le diría a la gente que confiara en la marca XXX }\end{array}$ \\
\hline $\begin{array}{c}\text { Evangelismo } \\
\text { (Matzler et al., 2007) }\end{array}$ & $\begin{array}{l}\text { 1. Podría ser un perfecto vendedor de la marca XXX } \\
\text { 2. Podría convertir a varios de mis amigos a la } \\
\text { marca XXX } \\
\text { 3. Podría convencer a otros de las cualidades de mi } \\
\text { vehículo marca XXX } \\
\text { 4. Les diría a todos que los vehículos de la marca } \\
\text { XXX están entre los más atractivos del mercado } \\
\text { 5. Si alguien tratara de desaprobar un vehículo } \\
\text { marca XXX, indudablemente le llamaría la atención }\end{array}$ \\
\hline $\begin{array}{l}\text { Promoción } \\
\text { (Kim et al., 2002; Yi y Gong, 2013; Shimul y Phau, } \\
\text { 2019) }\end{array}$ & $\begin{array}{l}\text { 1. Recomendaría un vehículo de la marca XXX a } \\
\text { otras personas para su compra/uso } \\
\text { 2. A partir de mi experiencia con la marca XXX le } \\
\text { hablaría bien de esta a otras personas } \\
\text { 3. Diría cosas positivas de la marca XXX } \\
\text { 4. Sugeriría a otras personas la compra/el uso de la } \\
\text { marca XXX } \\
\text { 5. Motivaría a mis amigos y familiares a que } \\
\text { compraran un vehículo de la marca XXX }\end{array}$ \\
\hline $\begin{array}{l}\text { Intención de recompra } \\
\text { (Chiu et al., 2009; Chai et al., 2015) }\end{array}$ & $\begin{array}{l}\text { 1. Probablemente volveré a comprar un vehículo } \\
\text { marca XXX } \\
\text { 2. Tengo la intención de comprar de nuevo un } \\
\text { vehículo marca XXX } \\
\text { 3. Es posible que en el futuro repita la compra de un } \\
\text { vehículo marca XXX } \\
\text { 4. Me gustaría volver a comprar un vehículo marca } \\
\text { XXX }\end{array}$ \\
\hline
\end{tabular}

